\title{
DE LO QUE NO HEMOS HABLADO: INFANCIA VULNERADA EN EL CONFLICTO ARMADO Por Semillero de Investigación Filoinfancia
}

\subsection{Un poco de historia}

Este texto se constituye en la reflexión del equipo, en torno a los hitos que se fueron dando en un proceso investigativo enfocado en menores de edad víctimas del conflicto armado en el departamento del Meta, y que coinciden parcialmente con los documentos de trabajo y avances de la investigación alojados en el repositorio institucional ${ }^{1}$, con el informe entregado al Centro de Investigación de la Universidad Santo Tomás y el resumen de una ponencia presentada en la III Bienal iberoamericana de infancias y juventudes ${ }^{2}$. Estos documentos obedecen a los avances que se iban suscitando con el desarrollo de la investigación desde 2017 hasta 2019. También se encuentran coincidencias con los títulos de eventos y resúmenes que existen en los aplicativos CvLAC de Minciencias.

Colombia durante los últimos años ha tenido un panorama semejante a la metamorfosis, en donde diversos aspectos del país se han transformado al ser permeados por el proceso de paz. Estas transformaciones han causado que la sociedad colombiana se polarice en posturas a favor o en contra del proceso de paz, aludiendo a categorías como verdad, justicia y reconciliación; como ya se conoce, este panorama surge desde el interés de algunas personas de continuar en el conflicto armado, tomando los medios como la catapulta para llegar a la población y causar un ambiente de desinformación y confusión.

Teniendo en cuenta esto, el grupo de investigación Nakota, de la Universidad Santo Tomás, se ha comprometido con el proceso de paz, a través de acciones de investigación en los que se entretejen relaciones con estudiantes universitarios y menores de edad que asisten a colegios públicos, docentes universitarios y de instituciones educativas de básica, así como directivas de los planteles y padres de familia. Todo ello se pretende por medio de ambientes democráticos que propicien la construcción de la ciudadanía. Las herramientas

${ }^{1}$ Disponible en https://repository.usta.edu.co/handle/11634/21942

${ }^{2}$ Disponible en https://repository.usta.edu.co/handle/11634/18706? cv=1\&show=full 
fundamentales se han tomado de la filosofía para niños $-\mathrm{FpN}$ - con su metodología predilecta de comunidades de diálogo.

Esto se ha logrado gracias al arduo recorrido en el que se han gestionado proyectos, seminarios, talleres, conversatorios y foros que tienen la mirada puesta en la construcción de paz. Este interés se manifestó desde el 2013, cuando el Semillero de investigación Lego empezó su construcción de un trabajo hacia la búsqueda del pensamiento filosófico en el contexto escolar, tomando como principal autor al filósofo colombiano Diego Pineda, quien ofrecía una innovadora propuesta de en $\mathrm{FpN}$ teniendo como pilar fundamental el desarrollo del pensamiento crítico a partir de las comunidades de diálogo.

A partir de lo anterior se desarrolló el seminario La filosofía para niños como estrategia para la comprensión y el pensamiento crítico en las instalaciones de la Universidad Santo Tomás, el 18 de octubre de 2013, cuyo ponente principal fue el profesor Diego Antonio Pineda. Este espacio permitió analizar los vínculos entre educación y pensamiento crítico en clave de FpN y de allí, surgió la iniciativa de organizar una red de promotores del pensamiento crítico, integrada por los profesores de filosofía del Meta, en su mayoría de corriente humanista, destacados por su pasión y compromiso en el desarrollo educativo.

Desde 2015 se consolida un plan de trabajo interdisciplinario que buscaba impactar en el contexto regional. En este punto, se empezó a enfocar el trabajo investigativo en los campos de Currículo y PEI, Literatura Vivencial y la implementación de la estrategia de Filosofía para niños, esas fueron las primeras líneas del semillero Filosofía e Infancia con el propósito claro en dirección a la construcción de paz.

Es así, como a partir de 2016 se genera un vínculo de cooperación investigativa con el semillero Infancia y Filosofía de la Universidad de los Llanos, UNILLANOS, con el cual se buscó fortalecer el proceso investigativo y formativo, realizando un trabajo colaborativo entre los integrantes de las dos instituciones. En este contexto se desarrollaron dos eventos importantes ese mismo año, el primero denominado La escuela infantil: territorios constructores de paz, en el auditorio Eduardo Carranza de Unillanos, sede Barcelona, en donde fue ponente el Mg. Luis Francisco Guerra, evento en el que se logró generar una 
reflexión a partir de los planteamientos de la infancia en torno a temas como guerra, paz, violencia y perdón.

Posteriormente se desarrolló el seminario FpN una estrategia hacia la construcción de la paz, en el mes de octubre. Se invitó como ponente a la Doctora Angélica Lucas Sátiro, quien dirige el proyecto Noria de Barcelona, España; también estuvieron como ponentes, el Mg. Víctor Andrés Rojas de la Universidad Minuto de Dios -UNIMINUTO- y Monseñor Oscar Urbina Ortega, arzobispo de la Arquidiócesis de Villavicencio. El propósito del seminario fue demostrar la importancia de la reflexión filosófica de la infancia como fundamento para la construcción de paz.

Es así, como se parte de la idea que en las comunidades de diálogo, se discuten opiniones, argumentos e informaciones, en ambientes de racionalidad y reflexión crítica. Esto implica en el ámbito de la educación, transformar las tradicionales jornadas de clase en espacios de diálogo que permitan la construcción de pensamientos razonables y flexibles frente a las realidades complejas del mundo de la vida (Lipman, 2004).

Dentro del trabajo realizado llamó la atención la no inclusión de la infancia en las primeras etapas de negociaciones de paz por parte del gobierno nacional y las FARC, el cual se fue involucrado al transcurrir de los diálogos, al evidenciarse por parte de la defensoría del pueblo la afectación de un número de catorce mil menores de edad al participar activamente en el desarrollo de estos grupos armados, estadísticas que se hacían latentes en la zona de los llanos orientales.

Esta realidad llevó a problematizar el ejercicio del quehacer docente, el analizar el currículo implementado en las escuelas y evaluar si el sistema educativo estaba preparado para afrontar la realidad. Si bien las entidades territoriales del Meta y Villavicencio dieron a conocer políticas públicas con el fin de atender esta población, aún se hacía necesario que la escuela construyera paz.

En 2017, el semillero Filosofía e Infancia postula el proyecto Infancia constructora de paz en la convocatoria del Fondo de Investigación -FODEIN-, de la Universidad Santo Tomás. El proyecto se desarrolló con estudiantes de los colegios como Departamental La Esperanza y el Colegio Catumare que se caracterizaban como víctimas del conflicto armado. El 
objetivo de la investigación era analizar el alcance de las comunidades de diálogo como estrategia para la construcción de paz a partir de la vivencia de las víctimas.

El proyecto se centró en la infancia, especialmente la que había sido víctima del conflicto armado interno, dado que la Oficina del alto comisionado (2015) consideraba que "la construcción de la paz es asunto de la sociedad en su conjunto que requiere de la participación de todos sin distinción" (p.1), aludiendo al concepto establecido en la ley 1448 de 2011, el cual señala que víctima es la persona que

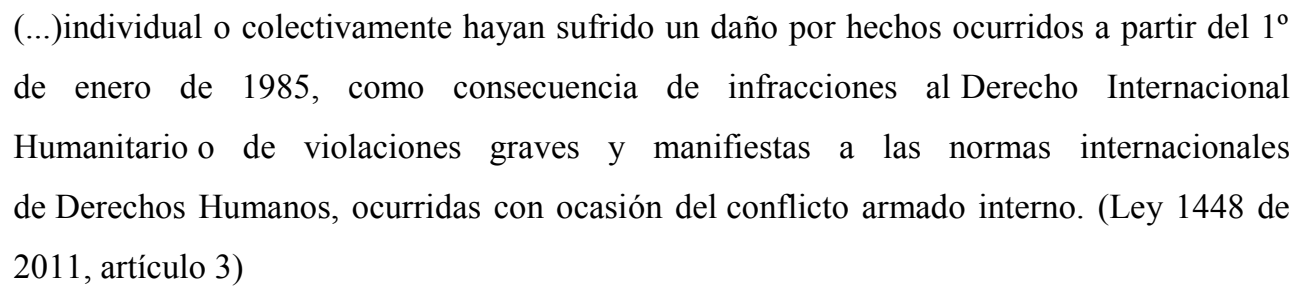

De manera preocupante, de las 8.604.210 víctimas del conflicto armado en Colombia, 97.436 estaban radicados en Villavicencio, tomando como fuente el Registro Único de Víctimas hasta diciembre de 2017. En el departamento del Meta fueron atendidas solamente 1.363 víctimas y de ellas, el 50,1\% (695 personas) migraron a Villavicencio. Para profundizar el panorama, se indicaba que había 86 casos de Vinculación de menores de edad a los grupos armados. Los indicadores de vínculo de menores de edad se hacían cada vez más alarmantes.

Por otra parte, la política pública de infancia en el proceso paz no se abordaba como era de esperarse, y por ello surge la inquietud de abordar desde la investigación las problemáticas que afrontaban los menores de edad víctimas del conflicto interno colombiano, en la ciudad de Villavicencio, a partir de las comunidades de diálogo y el pensamiento crítico. De esta manera el semillero de investigación podría contribuir en la memoria, en la búsqueda de sentido y en la transformación para lograr ambientes de paz.

Un hallazgo importante se logró a través de la caracterización de los niños y niñas que asistían al refuerzo escolar que ofrecía el Centro de Proyección Social en la comuna 8 de Villavicencio, pues se trataba de población desplazada a causa del conflicto interno; el refuerzo escolar es un espacio que ofrece la Universidad Santo Tomás como apoyo para el 
desarrollo de las actividades académicas a estudiantes de básica primaria o secundaria de instituciones educativas de la zona.

El equipo investigador se formulaba estas inquietudes ¿De qué manera la escuela contribuye a consolidar la paz? ¿Cómo puede la escuela abordar la historia del conflicto colombiano? ¿Qué puede hacer la escuela para evitar que los niños caminen por el sendero de la violencia? ¿Toman decisiones los niños y las niñas sin pensar? ¿Qué intereses llevan a involucrar a los niños en el conflicto colombiano? ¿Hay formas de prevenir la exclusión de los niños en el conflicto colombiano? Pero como el equipo estaba aplicando la metodología de Comunidades de diálogo en el refuerzo escolar, se formuló la pregunta ¿De qué manera las comunidades de diálogo se constituyen en una alternativa para afrontar las vivencias del conflicto armado en los estudiantes víctimas que asisten a los colegios La Esperanza y Catumare de Villavicencio?

De esta manera se buscaba analizar la repercusión de las comunidades de diálogo como alternativa para afrontar las vivencias del conflicto armado en los estudiantes víctimas que asistían a los colegios La Esperanza y Catumare de Villavicencio, puesto que eran los mismos menores que participaban en el programa de refuerzo escolar de la Universidad Santo Tomás.

Para recabar la información se diseñaron fichas de caracterización, el diario de campo, las comunidades de diálogo articuladas con entrevistas semiestructuradas y en profundidad. Ello permitió establecer un diagnóstico de la población infantil afectada por el conflicto armado que asistía a las instituciones educativas Catumare y Departamental La Esperanza de Villavicencio. A la vez se buscaba facilitar el intercambio reflexivo de comprensiones y significados al interior de las comunidades educativas involucradas en la investigación, con el fin de fortalecer el pensamiento crítico y en aras de la construcción de paz en sus ambientes escolares y sociales. El tercer objetivo consistía en beneficiar a las comunidades involucradas en el proyecto de investigación, a través de la realización de un taller en temas de humanismo y construcción de paz, con expertos nacionales e extranjeros.

Dentro del proceso de caracterización se encontró que los niños pertenecen a estratos socioeconómicos 0,1 y 2 , con edades entre 9 y 15 años, cuyos tutores, padres o cuidadores 
evidenciaban bajos niveles educativos. Las familias de los participantes en la investigación tenían entre 3 y 6 personas. Llamaba especialmente la atención que algunos participantes eran integrantes de población sorda y habían vivido la crueldad del conflicto en su infancia.

También se realizó la caracterización desde la perspectiva de los padres de familia de los integrantes del grupo de participantes, que vivieron la situación de conflicto de manera directa, en cuyas intervenciones manifestaron estar en contra de los estamentos y de los líderes gubernamentales, dado que no veían esperanza en la ley de víctimas y no veían con mayor expectativa las acciones del gobierno en defensa de las condiciones de vida digna, el acceso a la atención humanitaria, las políticas públicas de prevención, atención y reparación integral. Era población desplazada que veía el retorno a su lugar de origen como un imposible, y con igual dificultad el derecho a reubicarse en condiciones de voluntariedad, seguridad y dignidad. Las mujeres se mostraban desconcertadas frente a la esperanza de vivir libres de violencia intrafamiliar. En general, había un panorama de desesperanza frente a la ley 1448 de 2011 o ley de víctimas.

Las condiciones descritas en la citada ley permitieron adaptar los instrumentos de caracterización para poder construir el perfil de la población de víctimas del conflicto con la que se desarrolló la investigación. Ahora, de acuerdo con la Defensoría del Pueblo (2013) las características a las que hace referencia este perfil son aquellas personas que padecieron uno o varios de estos hechos de acto terrorista / Atentados / Combates / Enfrentamientos / hostigamientos, amenaza, desaparición forzada, desplazamiento forzado, secuestro, homicidio, tortura, delitos contra la libertad y la integridad sexual en desarrollo del conflicto armado, despojo y abandono de bienes, minas antipersonal / munición sin explotar y artefacto explosivo improvisado, vinculación de niños, niñas y adolescentes a actividades relacionadas con grupos armados.

En la caracterización de los participantes se siguió el perfil establecido por la ley de víctimas 1448 de 2011. Se descubre que 93,3\% de los hogares de los niños y niñas víctimas del conflicto, con quienes se desarrolló el proyecto Infancia constructora de paz, fueron afectados por abandono o despojo forzado de tierras; el 53,3\% por amenazas; el 33,3\% por actos terroristas, atentados, combates, enfrentamientos u hostigamientos; el 13,3\% por desaparición forzada; el 13,3\% por homicidio; el 13,3\% por masacre; el 6,7\% por tortura y 
el $6,7 \%$ por vinculación de menores de edad en actividades relacionadas con grupos armados, como se muestra en la gráfica número 1.

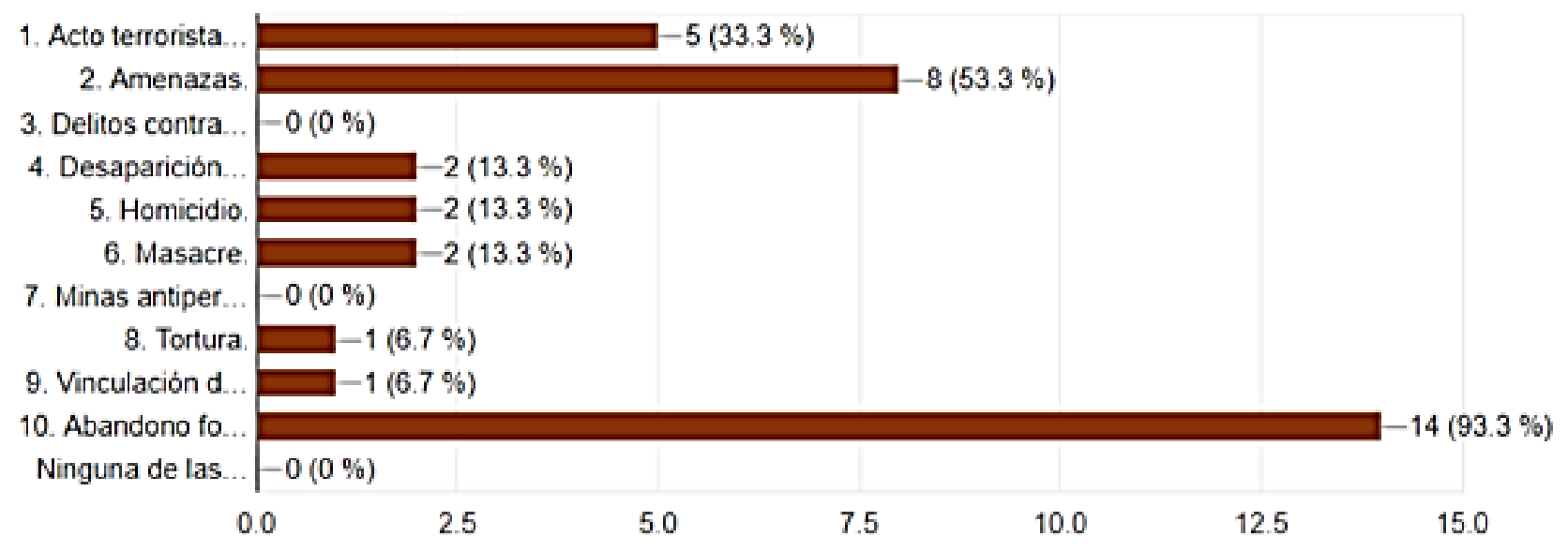

Gráfica número 1: Caracterización de víctimas en la fase I de la investigación

Fuente: archivo digital Grupo de Investigación Nakota

La caracterización en la fase II, con el grupo de familias del sector Playa Rica y Villa Lorena, de la comuna 8 de Villavicencio, indicaba que la mayoría había sido afectada por actos terroristas $(61,5 \%)$ y abandono forzado o despojo de tierras $(53,8 \%)$, entre otros factores que menciona la ley de víctimas, como se indica en la gráfica número 2.

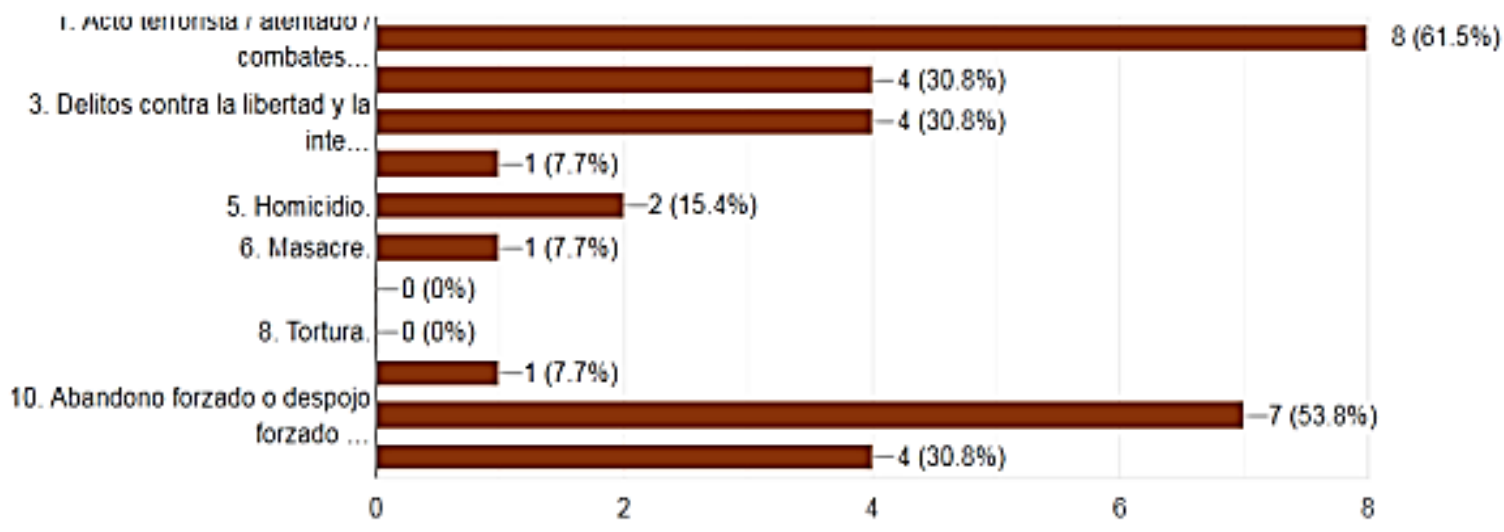

Gráfica número 2: Caracterización de víctimas en la fase II de la investigación Fuente: archivo digital Grupo de Investigación Nakota 
Tomando en cuenta el perfil de la comunidad investigada se encontró un vínculo de esta población con dificultades académicas en competencias de lectura, habilidades lógicas matemáticas e idiomas, de ahí que fue necesario fortalecer las acciones educativas, dado que los investigadores se habían propuesto beneficiar a la comunidad, en el marco del horizonte institucional de la USTA.

\subsection{En diálogo con los autores}

El grupo estaba de acuerdo en que la escuela debe enfatizar en su compromiso humanista con los menores de edad víctimas del conflicto armado y que formar lo humano implica abordar dimensiones espirituales, estéticas, cognitivas, el fortalecimiento de la voluntad y la búsqueda de sentido, lo cual se halla en autores como Mounier (1962), Delors (1996) y Morin (1999). La escuela así entendida es el escenario adecuado para realizar los propósitos de la humanidad, tal como se buscó desde el ideal griego de la paideia para la virtud o desde el espíritu humanista del renacimiento.

Siguiendo esa línea humanista en el trabajo con las comunidades vulnerables descritas, el equipo de investigación se encuentra con las categorías conceptuales como educación, comunidades de diálogo, paz y conflicto armado. Estas categorías se interrelacionaron, dándole una mirada a las cinco acepciones que sobre la paz considera la Real Academia Española, la primera de ellas como la situación en la que no existe lucha armada; la segunda como la armónica relación entre las personas, con comunidades libres de conflictos; la tercera, como el acuerdo entre las naciones para finalizar la guerra; la cuarta como la ausencia de ruido en un momento, y la quinta como el estado de quien está exento de perturbaciones por inquietudes o conflictos.

Manuel Kant (2001) aporta con respecto a las alianzas de paz, que ella no corresponde a estados de naturaleza, pues el estado natural es la guerra; las alianzas de paz implican un grado de confianza en que el enemigo tenga conciencia del pacto; cuanto esta no existe, se generan hostilidades que ineludiblemente conducen a guerra de exterminio y al alejamiento de la paz por la perpetuación de la guerra en la que se da la amenaza de hostilidad constante. "Por tanto, la paz es algo que debe ser instaurado" (p. 4) El mismo autor advierte 
que la paz no se logra solamente absteniéndose de romper las hostilidades, pues los integrantes de la comunidad se deben garantizar mutuas seguridades, como lo es propio de las sociedades civiles, y de esa manera no se ve al otro como enemigo potencial.

La dialéctica hegeliana aporta luces para entender la categoría conflicto, precisamente porque la vivencia de los conflictos representa la antítesis de las situaciones anheladas, las mismas que precedieron al conflicto y que ahora se desean. Esta antítesis es el sinsentido de la historia colombiana de 50 años de violenta guerra, en la que ha imperado el maltrato y el desconocimiento de la dignidad humana por parte de todos los actores. Pero ello convoca a un llamado generar acuerdos de paz hacia la construcción de una nueva sociedad, acuerdo que constituye la síntesis del esquema hegeliano. Este sencillo planteamiento no se refleja con la misma sencillez en el escenario de los hechos porque lograr la paz implica el abordaje de unos nexos históricos, emocionales y personales que han dejado huellas profundas en las personas catalogadas como víctimas del conflicto.

Con fortuna, se encuentra en la estrategia de las comunidades de diálogo y en los ejercicios de pensamiento crítico adecuadas herramientas que desde el ámbito educativo permitieron avanzar en esa dialéctica. Las comunidades de diálogo son el ambiente propicio para hacer manifiestas las contradicciones y liberar emociones, pensar desde la perspectiva de los otros y del sujeto mismo profundizar en diversos puntos de vista, lograr acuerdos y de esta manera asumir que se puede vivir en comunidad aunque existan desacuerdos.

A partir de las comunidades de diálogo se descubren, entonces elementos transformadores, relacionados principalmente con el diálogo. La educación se entiende como el escenario de igualdad incluyente, aspectos que son comunes con la pedagogía de la liberación y con la Teoría de la Acción Dialógica, pues los participantes pueden reclamar sus derechos y así cada persona, cada grupo y cada pueblo asume que puede buscar y vivir sus opciones. Esta realidad manifiesta la actividad pedagógica como proceso de plena comunicación y la educación como camino de la emancipación orientado a la formación integral del ser humano (Castellanos, 2013).

En efecto, las comunidades de diálogo constituyen una posibilidad terapéutica para resolver los conflictos, pues permiten limar asperezas y asumir las diferencias en los encuentros cara 
a cara, lo cual es favorable para sanar las heridas. El ejercicio terapéutico dio paso a la categoría resiliencia, que en la etnografía se cataloga como emergente. El proceso hace que las víctimas se sobrepongan a los agravios de la guerra, se acerquen de manera ineludible al tema del perdón, con lo cual se va asumiendo el rol resiliente de manera paulatina. Ese ejercicio se observó innumerables veces cuando los participantes narraban sus vivencias en las sesiones de trabajo.

Es importante anotar que este proceso no implica el olvido de los agravios, dado que el perdón no implica olvidar la ofensa, pero si un don de la víctima a su victimario como la oportunidad para cerrar ciclos de violencia (Echeburúa, 2013). Con el mismo autor, el equipo está de acuerdo en que la familia y la comunidad asumen un papel fundamental, toda vez que motivan a la víctima para que salga adelante y transforme su vida. De esta manera, quien fue víctima se habilita para sobreponerse a las adversidades y se convierte en generador de cambio personal y social.

Por su parte, los ejercicios de pensamiento crítico desarrollados en las comunidades de diálogo permitieron entender que la educación es escenario de emancipación, y con ella la pedagogía crítica como un saber enriquecido con este tipo de prácticas, lo cual traspasa las disciplinas, las interrelaciona y las ubica en el escenario de la realidad social para transformarla, a través del ejercicio formativo de las personas. De esta manera la pedagogía supera el carácter discursivo e impulsa al pedagogo, como sujeto reflexivo, "a entenderse como ser político transformador de las conciencias" (Gallego-Badillo, 1995. p. 30).

La Declaración Universal de los Derechos Humanos de 1948 consagra como objeto de la educación el pleno desarrollo de la personalidad humana, así como el fortalecimiento del respeto a los derechos humanos y a las libertades fundamentales. La declaración encarga a la educación la tarea de orientar la comprensión, la tolerancia y la amistad entre las naciones y los grupos étnicos o religiosos y la misión de promover "el desarrollo de las actividades de las Naciones Unidas para el mantenimiento de la paz" (Art. 26) Por ello es tan importante generar en las aulas ambientes donde se consolide el diálogo en todos los aspectos si se pretende que los estudiantes se formen para vivir en el mundo y para el 
mundo, para dar respuesta a las necesidades de su nación y consolidar una paz estable y duradera.

El proceso investigativo evidenció, como lo señalan diversos informes de Naciones Unidas, que los desplazados de zonas de conflicto se exponen a múltiples riesgos físicos y psicológicos, como quedó registrado en las fichas de caracterización aplicadas a los niños investigados e interpretadas a la luz de la ley 1448 de 2011. En efecto, no solo cargan en su memoria con el lastre de la amenazados y el peligro de los ataques, los cañones y las minas antipersona, sino que, ante el desplazamiento deben enfrentarse de manera forzosa a circunstancias nuevas en las ciudades, tales como la pobreza, la violencia urbana, el hambre y la insatisfacción de sus necesidades básicas.

Esta situación va generando conflictos internos en los niños, lo que hace urgente pensar en la necesidad de visualizar su realidad desde un ambiente de diálogo para generar alternativas, a través de ambientes que propicien el pensamiento crítico. De esta manera se rompe la cadena del maltrato y se orienta a las víctimas hacia un horizonte de paz y esperanza. Es por ello que por medio de la pedagogía liberadora se busca que esta sea la generación de educandos que construyan paz desde su entorno.

De otro modo, si no se ofrecen alternativas, las víctimas pueden optar por convertirse en actores del conflicto, continuando así el interminable ciclo de violencia que se ha vivido en el país a lo largo de más de cinco décadas y que no han dejado más que una nación fragmentada, con una economía muy débil y profundas heridas en la sociedad.

Al estudiar los contenidos de la citada ley 1448 de 2011, para nuestro caso, se encuentra que las víctimas en Colombia, tienen derecho a ser reparadas de manera adecuada, diferenciada, transformadora y efectiva por el daño que han sufrido, lo que incluye medidas de restitución, indemnización, rehabilitación, satisfacción y garantías de no repetición, en sus dimensiones individual, colectiva, material, moral y simbólica.

En esta investigación se evidenció que las vivencias del conflicto más frecuentes son el desplazamiento y reclutamiento forzado de conocidos y familiares; rechazo, prejuicios y marginación por parte de la sociedad. Como lo narró el entrevistado C702-4 cuando 
mencionó que las FARC reclutaron a la fuerza al segundo esposo de su mamá y duró un año "desaparecido".

Cuando hay actores armados en un territorio todo cambia, las relaciones están permeadas por el miedo y la desconfianza, que se constituyen a su vez en los elementos más importantes en la ruptura de relaciones sociales, situación que se evidencia en el relato del participante S803 “(...) Cambiar de un lugar a otros tuvimos que empezar una vida nueva, conocer personas cambiar de colegio y de iglesia fue un cambio radical. Por otro lado, la entrevistada C802-32 menciona sobre la misma situación de desplazamiento por causa del conflicto las "secuelas de lo que pasó en mi familia, entraron a la casa y despojaron a mi familia y eso me dejó marcada y un poco traumada. (...).

El encuentro con las nuevas condiciones de vida y de socialización trae consigo nuevos procesos de adaptación en los niños y jóvenes víctimas del conflicto. La carga emocional crea una barrera que no permite el correcto interactuar en grupo, sentimientos de miedo, tristeza, ansiedad y demás que hacen mella en la autoestima, se conjugan con los prejuicios de aquellos que no han vivido el conflicto y dan un significado a la figura de la víctima como una persona en condición de desventaja o como un individuo que puede significar una amenaza en su entorno, de ahí que algunos participantes consideren que "estudiando, perdonando. mirar hacia un futuro, investigando... poder defender, hablar de paz" (C1032).

Las narraciones de las víctimas del conflicto armado permiten establecer las representaciones sociales en la mente de las víctimas y que son expresadas no solo de manera oral sino en todos los referentes simbólicos de nuestra sociedad que se transmiten en un proceso de exteriorización de emociones e historias. Cada relato, entonces, no solo evidencia el pasado, sino que recupera, junto con la historia, al propio protagonista con sus emociones, sentimientos, sensaciones, interpretaciones, superando a la vez, tanto los límites espacio-temporales como las representaciones construidas por otros acerca de la acción histórica de los hechos sociales ocurridos durante el conflicto armado en Colombia (Nieto, 2010). 
Ahora bien, el trabajo educativo en torno a estas vivencias, supera la remembranza que podría tornarse en revictimización. En efecto se emprenden acciones educativas para fortalecer la resiliencia. En los diarios de campo se registraba cómo los menores de edad iban mostrando actitudes resilientes, a medida que encontraban un apoyo externo y compañía de otras personas que aportaban al crecimiento personal de los menores de edad. Las comunidades de diálogo que se aplicaron se enriquecieron con entrevistas en profundidad, juego de roles, integración deportiva, celebraciones, teatro, entre otras estrategias propias del campo educativo.

El impacto de ese trabajo de intervención educativa tiene una mirada externa en el estudio adelantado por la Universidad Minuto de Dios y la Universidad de Antioquia, publicado en la revista Praxis y Saber, en el que se expresa, refiriéndose al proyecto Infancia constructora de paz, del CAU Villavicencio, que los niveles de "resiliencia, después de las comunidades de diálogo, fueron superiores que los puntajes si no se hubiera llevado a cabo la intervención" (Alvarán, S. et al.2019, p. 147). De tal forma que se aprecia el impacto positivo en favor de la resiliencia en víctimas del conflicto armado, visto desde la óptica de los menores de edad, desde los investigadores y se confirma con el estudio externo. Este dato también está publicado ${ }^{3}$. $\begin{array}{cc}\text { Disponible } & \text { en } \\ 01592019000200139 \& \text { script }=\text { sci arttext }\end{array}$

http://www.scielo.org.co/scielo.php?cv=1\&pid=S2216- 


\section{REFERENCIAS}

Alto comisionado para la paz (2015). Borrador conjunto. Participación política: apertura democrática para construir la paz. Bogotá, Colombia: Oficina del alto comisionado para la paz.

Alvarán-López, S. M., Carrero-Torres, C. T., Castellanos-Triviño, H. R., \& Pinilla-López, H. Y. (2019). Resiliencia infantil y pensamiento multidimensional como factor de transformación social. Praxis \& Saber, 10(23), 139-156.

doi:10.19053/22160159.v10.n23.2019.9727

Castellanos, H. (2013) Fortalecimiento del Pensamiento crítico en la educación básica a través de las comunidades de diálogo [Trabajo investigativo de maestría. Universidad de Caldas].

Centro Nacional de Memoria Histórica. (2015). Una nación desplazada. CNMH.

Congreso de la República de Colombia. (2011). Ley 1448 por medio de la cual se dictan medidas de atención, asistencia y reparación integral a las víctimas del conflicto armado interno. Bogotá, Colombia.

Defensoría del pueblo de Colombia. (2013). Algunas cosas que debes saber sobre la Ley de Víctimas. Bogotá, Colombia.

Delors, J. (1996). La educación encierra un tesoro. Madrid: Santillana.

Echeburúa, E. (2013). El valor psicológico del perdón en las víctimas y en los ofensores. Eguzkilore.

Gallego-Badillo, R. (1995). Saber pedagógico, una visión alternativa. Editorial Magisterio.

Kant, I. (2001). La paz perpetua. Madrid, España: Ediciones Mestas.

Lipman, M.(2004). Natasha: aprender a pensar con Vygotsky. Barcelona, Editorial: Gedisa. 
Morin, E. (1999). Los siete saberes necesarios para la educación del futuro. Francia: UNESCO.

Mounier, E. (1962). El personalismo (No. 141.144). Eudeba.

Nieto, Patricia. (2010). Relatos autobiográficos de víctimas del conflicto armado: una propuesta teórico-metodológica. Revista de Estudios Sociales, número 3.

ONU (1948). Declaración universal de los derechos humanos. Ginebra: Organización de las Naciones Unidas. 